\title{
A new calculation technique of characteristics of multichannel measurer
}

\author{
O. V. Chernoyarov ${ }^{1}$, M. Vaculik ${ }^{2}$, A. E. Rozanov ${ }^{1}$ \\ ${ }^{1}$ Dept. of Radio engineering Devices of the National Research University “MPEI”, Moscow, Russia \\ ${ }^{2}$ Dept. of Telecommunications and Multimedia of the University of Zilina, Zilina, Slovakia
}

\section{Email address:}

o_v_ch@mail.ru(O.V. Chernoyarov), martin.vaculik@fel.uniza.sk(M.. Vaculik), nigilus@mail.ru(A. E. Rozanov)

\section{To cite this article:}

O. V. Chernoyarov, M. Vaculik, A. E. Rozanov. The New Calculation Technique of Characteristics of Multichannel Measurer. Science Journal of Circuits, Systems and Signal Processing. Vol. 2, No. 3, 2013, pp. 78-85. doi: 10.11648/j.cssp.20130203.11

\begin{abstract}
A new calculation technique of accuracy characteristics of multichannel measurer was offered, ensuring essentially more exact results in comparison with the known ones. The expressions for the signal parameter maximum likelihood estimate bias and variance were found, in case of the multichannel reception of the quasidetermined or random signal and while the prior distribution of the informative parameter and the number of channels can be any. The influence of the value of the prior interval length and the number of channels on the accuracy of this estimate was investigated. The procedures were specified of the minimal channel number choice with the predetermined error of measurement. By statistical modeling methods the range of applicability of the theoretical formulae was established for the estimate characteristics, with the different numbers of channels and signal-to-noise ratios.
\end{abstract}

Keywords: Multichannel Reception, Maximum Likelihood Estimate, Measurement Characteristics, Statistical Modeling

\section{Introduction}

The optimal (according to a maximum likelihood method) signal parameter measurer receiving a realization of observable data in a kind of signal and hindrance sum generates the output effect which is proportional to the functional of likelihood ratio (FLR) or its logarithm [1, 2]. It is supposed that values of output effect are formed within the whole prior definition interval $\left[L_{1}, L_{2}\right]$ of the estimated parameter $1_{0}$. As a result, $1_{m}$ the position of an absolute maximum of $M(1)$, logarithm of FLR is an estimate that should be determined. However it is seldom possible to make the optimal measurer with continuous change of current parameter value. In most cases it is necessary to resort to the multichannel scheme with discrete like values of unknown parameter [2, 3].

In case of the multichannel scheme the measurer yields $M(1)$ samples in $v$ equidistant points $1_{i}, i=1,2, \ldots, v$ of the prior interval $\left[L_{1}, L_{2}\right]$. As an estimate the value $l_{k}$ is accepted, corresponding to the channel with the greatest output signal.

Statistical characteristics of an estimate $1_{k}$ of the signal parameter $1_{0}$ are considered in $[2,3]$, when the measurer is used with the odd number of channels. It was assumed that the output signal-to-noise ratio (SNR) does not depend on value $l_{0}$ (i.e. the parameter $l_{0}$ is nonpower), and prior distribution of the estimated parameter $1_{0}$ obeys to the uniform law. However, the theoretical formulae received there for bias (systematic error) and variance (mean square error) of multichannel estimate leave out of account all possible conditions and results of measurements, and thereof they may produce a major error. Besides, there is a big class of signals and unknown parameters for which assumptions mentioned above are not carried out. Also the number of channels in the measurer can be both odd and even. Thereby correction and generalization of the results [2,3] are of interest, in case of the estimate of the arbitrary (power or nonpower) signal parameter $1_{0}$ with the prior distribution $w_{p r}\left(1_{0}\right)$, generally distinct from the uniform, if the multichannel scheme with any number of channels is used.

\section{Characteristics of the Signal Parameter Estimate In Case of the Multichannel Reception}

Let us suppose that the optimal (maximum likelihood) 
estimate $1_{m}$ (in the receiver with a continuous change of a current value of the measurable parameter $1_{0}$ ) is described by the probability density $w\left(1_{m} \mid 1_{0}\right)$. We designate the number of the channel, nearest to $\mathrm{l}_{0}$, as $\mathrm{n}$, and the distance between two adjacent channels as $\Delta=1_{i}-1_{i-1}$. Then with the fixed value of $1_{0}$ it is possible to present the conditional bias $b_{v}\left(1_{k} \mid 1_{0}\right)=\left\langle 1_{k}\right\rangle-1_{0}$ and variance $V_{v}\left(1_{k} \mid 1_{0}\right)=\left\langle\left(1_{k}-1_{0}\right)^{2}\right\rangle$ of the estimate $1_{k}$ in the multichannel measurer as follows:

$$
b_{v}\left(1_{k} \mid 1_{0}\right)=\sum_{i=-(n-1)}^{v-n}(i \Delta) P_{0 i}, V_{v}\left(1_{k} \mid 1_{0}\right)=\sum_{i=-(n-1)}^{v-n}(i \Delta)^{2} P_{0 i}
$$

Where

$$
P_{0 i}=P\left[1_{k}-n \Delta=L_{1}+(i-1 / 2) \Delta\right]=\int_{L_{1}+(n+i-1) \Delta}^{L_{1}+(n+i) \Delta} w\left(1_{m} \mid 1_{0}\right) d 1_{m},
$$

and \langle\rangle designates the averaging operation for all the possible realizations of the observable data.

If the parameter $1_{0}$ is described by the prior probability density $w_{p r}\left(1_{0}\right)$, then, similarly to Eq. (1), for the unconditional estimate bias $b_{v}\left(1_{k}\right)$ and variance $V_{v}\left(1_{k}\right)$, we have

$$
b_{v}\left(1_{k}\right)=\sum_{i=-(v-1)}^{v-1}(i \Delta) P_{i}, \quad V_{v}\left(1_{k}\right)=\sum_{i=-(v-1)}^{v-1}(i \Delta)^{2} P_{i} .
$$

Here

$$
\begin{gathered}
P_{i}=\sum_{n=1}^{v} P\left[\mathrm{l}_{k}-n \Delta=L_{1}+(i-1 / 2) \Delta\right]= \\
=\sum_{n=1}^{v} \int_{L_{1}+(n-1) \Delta}^{L_{1}+n \Delta} w_{p r}\left(1_{0}\right) \int_{L_{1}+(n+i-1) \Delta}^{L_{1}+(n+i) \Delta} w\left(1_{m} \mid 1_{0}\right) d \mathrm{l}_{m} d \mathrm{l}_{0} .
\end{gathered}
$$

Formulae (2) become essentially simpler, if the probability density $w\left(1_{m} \mid 1_{0}\right)$ of the maximum likelihood estimate (MLE) $1_{m}$ depends on a difference of arguments: $w\left(1_{m} \mid 1_{0}\right)=w\left(1_{m}-1_{0}\right) \quad$ - i.e. the parameter $1_{0}$ is nonpower $[1,2]$, and the prior distribution $w_{p r}\left(1_{0}\right)$ is described by the uniform distribution law: $w_{p r}\left(1_{0}\right)=1 / v \Delta$. Then for the probability $P_{i}$ there can be written down

$$
P_{i}=\frac{1}{\Delta} \int_{0}^{\Delta}[F(x+i \Delta)-F(x+(i-1) \Delta)] d x
$$

Where $F(x)=\int_{L}^{x} w\left(1_{m}\right) d \mathrm{l}_{m}$ is the distribution function of MLE $1_{m}$.

Substituting Eq. (3) in the Eq. (2) results in

$$
b_{v}\left(1_{k}\right)=\sum_{i=-(v-1)}^{v-1} i\left[\int_{i \Delta}^{(i+1) \Delta} F(x) d x-\int_{(i-1) \Delta}^{i \Delta} F(x) d x\right] \text {, }
$$

$$
V_{v}\left(1_{k}\right)=\Delta \sum_{i=-(v-1)}^{v-1} i^{2}\left[\int_{i \Delta}^{(i+1) \Delta} F(x) d x-\int_{(i-1) \Delta}^{i \Delta} F(x) d x\right] .
$$

If the distribution of MLE $1_{m}$ is symmetric concurrently, i.e. $F(x)=1-F(-x)$, then the estimate $1_{k}$ in the multichannel measurer is unbiased $-b_{v}\left(1_{k}\right)=0$, and its variance is

$$
V_{v}\left(1_{k}\right)=2 \Delta \sum_{i=1}^{v-1} i^{2} \int_{0}^{\Delta}[F(x+i \Delta)-F(x+(i-1) \Delta)] d x
$$

Under conditions of high posterior accuracy of MLE $1_{m}$, when the digitization errors are comparable with the potential ones, caused by the hindrance effect, it is possible to confine ourselves to the first summand in the sum (4). We designate the variance of MLE $1_{m}$ as $\sigma_{0}^{2}$. Then, considering that $F(x+\Delta) \approx 1$ under $\Delta \gg \sigma_{0} \quad\left(\Delta \geq 2 \div 3 \sigma_{0}\right)$, and extending the top limit of integration to infinity, we find the known result $[2,3]$ :

$$
V_{v}\left(1_{k}\right) \cong 2 \Delta \int_{0}^{\infty}[1-F(x)] d x
$$

Let us now consider the applicability of the formulae (2) in the practical applications.

\section{Estimate of Rectangular Video Pulse Duration}

Let an additive mix

$$
x(t)=s\left(t, \tau_{0}\right)+n(t)
$$

Be received by the measurer input.

$$
s\left(t, \tau_{0}\right)= \begin{cases}a, & 0 \leq t \leq \tau_{0}, \\ 0, & t<0, t>\tau_{0},\end{cases}
$$

Here is the useful signal with amplitude and duration $\tau_{0}$, while $n(t)$ is Gaussian white noise with one-sided spectral density $N_{0}$. With the observable realization (5), it is necessary to estimate the parameter $\tau_{0}$, which values are from the prior interval $\left[\mathrm{T}_{1}, \mathrm{~T}_{2}\right]$.

In compliance with $[1,2]$, we write down the logarithm of FLR in terms of

$$
M(\tau)=\frac{2 a}{N_{0}} \int_{0}^{\tau} x(t) d t-\frac{a^{2} \tau}{N_{0}}, \tau \in\left[\mathrm{T}_{1}, \mathrm{~T}_{2}\right]
$$

Then the estimate $\tau_{k}$ of the duration $\tau_{0}$ of the pulse (6) in the maximum likelihood measurer with $v$ channels, is now defined as

$$
\tau_{k}=\arg \sup M\left(\tau_{i}\right), i=1,2, \ldots, v .
$$

Here $\tau_{i}=\mathrm{T}_{1}+(i-1 / 2) \Delta$ is the pulse duration, to which $\mathrm{i}$-th channel is tuned, and $\Delta=\left(\mathrm{T}_{2}-\mathrm{T}_{1}\right) / v$ is the above stated 
distance between the two adjacent channels.

According to [4], the probability density of $\operatorname{MLE} \tau_{m}=\arg \sup M(\tau), \tau \in\left[\mathrm{T}_{1}, \mathrm{~T}_{2}\right]$ in the measurer with the continuous change of the current value of the estimated parameter can be represented as

$$
\begin{aligned}
& w\left(\tau_{m} \mid \tau_{0}\right)=f\left(\frac{z_{s}^{2}\left|\tau_{m}-\tau_{0}\right|}{2 \tau_{s}}\right) / \int_{\mathrm{T}_{1}}^{\mathrm{T}_{2}} f\left(\frac{z_{s}^{2}\left|\tau_{m}-\tau_{0}\right|}{2 \tau_{s}}\right) d \tau_{m} \\
& f(x)=\Phi(\sqrt{x / 2})-1+\exp (2 x)[1-\Phi(3 \sqrt{x / 2})]
\end{aligned}
$$

Where $z_{s}^{2}=2 a^{2} \tau_{s} / N_{0}$ is the output SNR for middle pulse duration $\tau_{s}=\left(\mathrm{T}_{1}+\mathrm{T}_{2}\right) / 2$, and

$\Phi(x)=\int_{-\infty}^{x} \exp \left(-t^{2} / 2\right) d t / \sqrt{2 \pi}$ is the probability integral.

Then characteristics of the estimate (8) for the specified prior probability density $w_{p r}\left(\tau_{0}\right)$ of the parameter $\tau_{0}$ can be found from Eq. (2), with $w_{p r}\left(\tau_{0}\right)$ and $w\left(\tau_{m} \mid \tau_{0}\right)$ (9) substituting $w_{p r}\left(1_{0}\right)$ and $w\left(1_{m} \mid 1_{0}\right)$, respectively.

In Figs. 1, 2 the theoretical dependences are shown of the normalized variance $\tilde{V}_{v}=V_{v}\left(\tau_{k}\right) / \tau_{s}^{2}$, calculated by the formulae (2), (9), with

$$
w_{p r}\left(\tau_{0}\right)=1 /\left(\mathrm{T}_{2}-\mathrm{T}_{1}\right)
$$

and the prior interval length value of either $\left(\mathrm{T}_{2}-\mathrm{T}_{1}\right) / \tau_{s}=1$ (Fig. 1), or $\left(\mathrm{T}_{2}-\mathrm{T}_{1}\right) / \tau_{s}=2$ (Fig. 2). Here the curves 1 correspond to $v=2 ; 2-v=3 ; 3-v=6$.

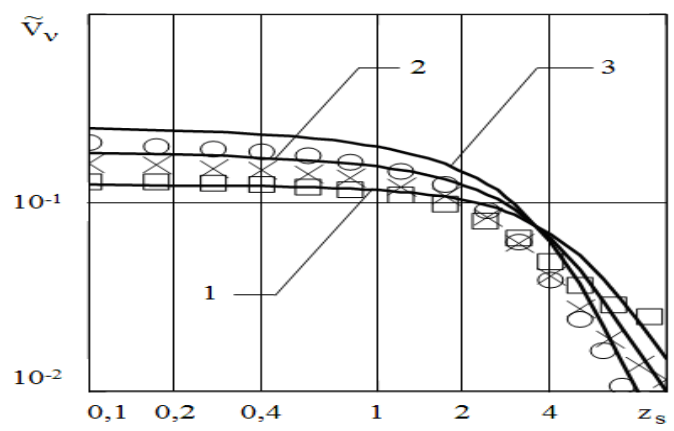

Figure 1. Normalized variance of duration estimate of rectangular video pulse under $\left(T_{2}-T_{1}\right) / \tau_{s}=1$

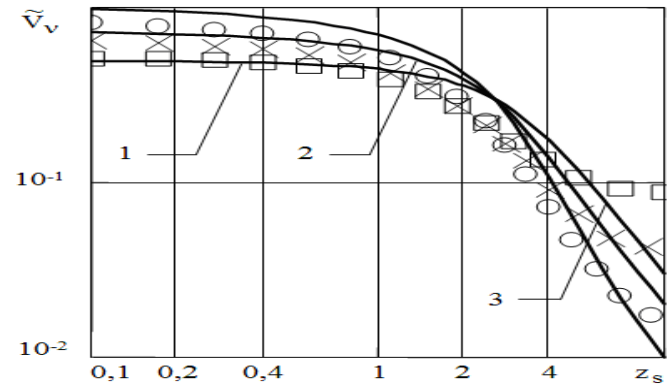

Figure 2. Normalized variance of duration estimate of rectangular video pulse under $\left(T_{2}-T_{1}\right) / \tau_{s}=2$.

Experimental characteristics of the estimate (8) were found by means of statistical computer modeling. During the modeling process, the samples $M\left(\tau_{i}\right)$ of the functional $M(\tau)$ (7) were formed, from the prior interval $\left[\mathrm{T}_{1}, \mathrm{~T}_{2}\right]$ and with the specified values of SNR $z_{s}$ and durations $\tau_{0}, \tau_{i}$ :

$$
M\left(\tau_{i}\right)=z_{s}^{2}\left[\min \left(\tilde{\tau}_{i}, \tilde{\tau}_{0}\right)-\tilde{\tau}_{i} / 2\right]+z_{s} W_{\tilde{\tau}}\left(\tilde{\tau}_{i}\right)
$$

Here $\tilde{\tau}_{i}=\tau_{i} / \tau_{s}, \tilde{\tau}_{0}=\tau_{0} / \tau_{s}$, and $W_{\tilde{\tau}}\left(\tilde{\tau}_{i}\right)$ is the sample of the standard Wiener process with zero mathematical expectation and dispersion $\tilde{\tau}_{i}$.

Samples of the Wiener process $W_{\tilde{\tau}}\left(\tilde{\tau}_{i}\right)$ in Eq. (11) were obtained as [5]

$$
\begin{cases}W_{\tilde{\tau}}\left(\tilde{\tau}_{i}\right)=\alpha_{1} \sqrt{\tilde{\tau}_{1},} & i=1, \\ W_{\tilde{\tau}}\left(\tilde{\tau}_{i}\right)=W_{\tilde{\tau}}\left(\tilde{\tau}_{i-1}\right)+\alpha_{i} \sqrt{\tilde{\tau}_{i}-\tilde{\tau}_{i-1}}, & i>1,\end{cases}
$$

Where $\alpha_{i}$ are independent Gaussian random numbers with zero mathematical expectations and unit dispersions? Formation of Gaussian numbers $\alpha_{i}$ with parameters $(0,1)$ was carried out in terms of the independent random numbers $\vartheta_{n}$, uniformly distributed within the interval $[0,1]$ by the Cornish-Fisher method [6]:

$$
\alpha_{i}=\mathrm{A}_{i}+\frac{\mathrm{A}_{i}^{3}-3 \mathrm{~A}_{i}}{20 N}, A_{i}=\sqrt{\frac{12}{N}} \sum_{n=1}^{N}\left[\vartheta_{N(i-1)+n}-0,5\right] .
$$

As follows from [6], the number of summands $\mathrm{N}$ in the sum (13) was chosen equal to 5.

For each realization of $M(\tau)$, generated by Eqs. (11)-(13), both the estimate $\tau_{k}$ was determined according to Eq. (8) and its characteristics were calculated. It was supposed that duration $\tau_{0}$ of the useful signal (6) in each testing was a uniformly distributed random value with a probability density (10). In Figs. 1, 2 experimental dependences of the variance $\tilde{V}_{v}$ for $v=2$ (squares), $v=3$ (crosses) and $v=6$ (circles) are presented, obtaining from the statistical modeling. Each experimental $\tilde{V}_{v}$ value was found as a result of the processing of at least $10^{5}$ realizations of $M(\tau)$. Consequently, confidence intervals boundaries deviate from experimental values no more than up to $5 \ldots 10 \%$ with the probability of 0.9 .

According to Figs. 1, 2, the theoretical dependences for the variance of the multichannel duration estimate (8) approximate the experimental data in a satisfactory manner, at least, for $z_{s} \geq 0,1, \quad v \geq 2$.

\section{Estimate of Time and Power Parameters of Random Radio Pulse}

Let us suppose now that the useful signal received against white noise $n(t)$ is random radio pulse, so that realization of the observable data has the appearance.

$$
x(t)=s\left(t, \lambda_{0}, \tau_{0}, D_{0}\right)+n(t), t \in[0, T],
$$

Where 


$$
s\left(t, \lambda_{0}, \tau_{0}, D_{0}\right)=\xi(t) I\left(\frac{t-\lambda_{0}}{\tau_{0}}\right), I(x)= \begin{cases}1, & |x| \leq 1 / 2, \\ 0, & |x|>1 / 2,\end{cases}
$$

$\lambda_{0}$ Is time delay, $\tau_{0}$ is duration of the pulse, and $\xi(t)$ is centered stationary Gaussian random process possessing spectral density

$$
G(\omega)=\frac{\pi D_{0}}{\Omega}\left[I\left(\frac{\theta-\omega}{\Omega}\right)+I\left(\frac{\theta+\omega}{\Omega}\right)\right]
$$

In Eq. (16) it is designated: $\theta$ - band center, $\Omega$ - bandwidth and $D_{0}-$ dispersion of the process $\xi(t)$.

With the observable realization (14) it is necessary to estimate unknown parameters $\lambda_{0} \in\left[\Lambda_{1}, \Lambda_{2}\right], \tau_{0} \in\left[\mathrm{T}_{1}, \mathrm{~T}_{2}\right]$, $D_{0} \in[0, \infty)$. It is assumed that the condition $\Lambda_{1}-\mathrm{T}_{2} / 2 \leq \lambda_{0}-\tau_{0} / 2<\lambda_{0}+\tau_{0} / 2 \leq \Lambda_{2}+\mathrm{T}_{2} / 2$ is satisfied, so the pulse (15) is always situated within the observation interval $[0, T]$.

We now consider a practically important case when the process $\xi(t)$ fluctuations are "fast", i.e. $\mu=\tau_{0} \Omega / 2 \pi \gg 1$. Then MLEs $\lambda_{m}, \tau_{m}, D_{m}$ of time delay $\lambda_{0}$, duration $\tau_{0}$ and dispersion $D_{0}$ are defined as [7]

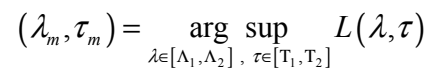

$$
\begin{aligned}
& D_{m}=\max \left[0 ; M\left(\lambda_{m}, \tau_{m}\right) / \tau_{m}-E_{N}\right]
\end{aligned}
$$

Here

$$
\begin{array}{r}
L(\lambda, \tau)=\tau E_{N}\left\{M(\lambda, \tau) / \tau E_{N}-\ln \left[M(\lambda, \tau) / \tau E_{N}\right]-1\right\} \\
M(\lambda, \tau)=\int_{\lambda-\tau / 2}^{\lambda+\tau / 2} y^{2}(t) d t \quad y(t)=\int_{-\infty}^{\infty} x\left(t^{\prime}\right) h\left(t-t^{\prime}\right) d t
\end{array}
$$

Is observable realization (14) response of the filter with transfer function $H(\omega)$, satisfying a condition $|H(\omega)|^{2}=I[(\theta-\omega) / \Omega]+I[(\theta+\omega) / \Omega] \quad, \quad$ and $E_{N}=\Omega N_{0} / 2 \pi$ is a signal band mean noise power.

It is easy to see that the measurer (17) can be practically realized only as a multichannel device, so that the estimates $\lambda_{k}, \tau_{k} D_{k}$ of parameters $\lambda_{0}, \tau_{0} D_{0}$ are defined as

$$
\begin{gathered}
\tau_{k}=\underset{\tau_{i}}{\arg \sup }\left[\sup _{\lambda \in\left[\Lambda_{1}, \Lambda_{2}\right]} L(\lambda, \tau)\right], \lambda_{k}=\underset{\lambda \in\left[\Lambda_{1}, \Lambda_{2}\right]}{\arg \sup } L\left(\lambda, \tau_{k}\right) \\
D_{k}=\max \left[0 ; M\left(\lambda_{k}, \tau_{k}\right) / \tau_{k}-E_{N}\right]
\end{gathered}
$$

Where $\tau_{i}=\mathrm{T}_{1}+(i-1 / 2) \Delta, \Delta=\left(\mathrm{T}_{2}-\mathrm{T}_{1}\right) / v, i=\overline{1, v}$ and $v$ is the number of the measurer channels.

Let us consider characteristics of joint estimates (19). We designate

$$
|\delta|=\left(\mathrm{T}_{2}-\mathrm{T}_{1}\right) / 2 \tau_{s}=\left(\mathrm{T}_{2}-\mathrm{T}_{1}\right) /\left(\mathrm{T}_{2}+\mathrm{T}_{1}\right)
$$

for the signal duration (15) maximum possible absolute deviation from the average duration $\tau_{s}=\left(\mathrm{T}_{2}+\mathrm{T}_{1}\right) / 2$. Then, in terms of the results received in [8] for the maximum variance of time delay estimate $\lambda_{k}$, we can state that

$$
\begin{gathered}
V\left(\lambda_{k} \mid \lambda_{0}\right)=P_{0} V_{d}\left(\lambda_{k} \mid \lambda_{0}\right)+ \\
+\left(1-P_{0}\right)\left[\left(\Lambda_{1}^{2}+\Lambda_{1} \Lambda_{2}+\Lambda_{2}^{2}\right) / 3-\lambda_{0}\left(\Lambda_{1}+\Lambda_{2}\right)+\lambda_{0}^{2}\right] .
\end{gathered}
$$

Here

$$
\begin{gathered}
V_{0}\left(\lambda_{k} \mid \lambda_{0}\right)=\left(\tau_{s}^{2} / 3\right)\left\{3 \delta_{v}^{2} / 8+H\left[z_{s} \sqrt{2 \psi(2-\psi) \delta_{v}}\right] \times\right. \\
\times\left[4 \psi(2-\psi)^{2}(4 \psi-5) z_{s}^{2} \delta_{v}^{3}+2(2-\psi)(5-3 \psi) \delta_{v}^{2}+\right. \\
\left.+1+3(16 \psi-29) \delta_{v} / \psi z_{s}^{2}+39 / \psi^{2} z_{s}^{4}\right]+\sqrt{(2-\psi) \delta_{v} / \pi \psi} \times \\
\left.\rtimes\left[2 \psi(2-\psi)(5-4 \psi) z_{s} \delta_{v}^{2}+(22 \psi-35) \delta_{v} / z_{s}+39 / \psi z_{s}^{3}\right]\right\}, \\
P_{0}=\frac{1}{\sqrt{2 \pi}} \int_{1}^{\infty} \exp \left[-\frac{\tilde{m} x}{\sqrt{2 \pi}} \exp \left(-\frac{x^{2}}{2}\right)\right]\left\{\sqrt{\frac{\tilde{m}_{S}\left(2-\tilde{m}_{S}\right)}{2 \pi} \zeta(x) \times}\right. \\
\left.\times \exp \left[-\frac{\zeta^{2}(x)}{2-\tilde{m}_{S}}\right]+\left[2+\tilde{m}_{S} \zeta^{2}(x)-\tilde{m}_{S}\right] \exp \left(-\frac{\zeta^{2}(x)}{2}\right)\right\} d x
\end{gathered}
$$

are the maximum variance and probability of a reliable estimate $\lambda_{k}$ respectively, and $z_{s}^{2}=\mu_{s} q_{0}^{2} /\left(1+q_{0}\right)^{2}$ is SNR for a pulse (15), the duration of which is equal to average duration $\tau_{s}$,

$$
\begin{gathered}
\mu_{s}=\tau_{s} \Omega / 2 \pi, q_{0}=D_{0} / E_{N}, H(x)=\exp \left(x^{2} / 2\right)[1-\Phi(x)], \\
\zeta(x)=\left[x-z_{s}\left(1+q_{0}\right)\left(1-\delta_{v}\right)\right] / \sqrt{1+q_{0}\left(2+q_{0}\right)\left(1-\delta_{v}\right)}, \\
\tilde{m}_{S}=\delta_{v} /\left[1+q_{0}\left(2+q_{0}\right)\left(1-\delta_{v}\right)\right], \quad \delta_{v}=|\delta| / v, \\
\psi=2\left(1+q_{0}\right)^{2} /\left[1+\left(1+q_{0}\right)^{2}\right], \quad \tilde{m}=\left(\Lambda_{2}-\Lambda_{1}\right) / \tau_{s} .
\end{gathered}
$$

As reliable estimate $\lambda_{k}[1,2]$, the estimate is understood, found on the assumption that $\left|\lambda_{k}-\lambda_{0}\right| \leq\left(\tau_{k}+\tau_{0}\right) / 2$, when mean value (signal function) of the sufficient statistics $M(\lambda, \tau)$ (18), with $\lambda=\lambda_{k}$ and $\tau=\tau_{k}$, is distinct from $\tau_{k} E_{N}$.

According to [7], the maximum variance of estimate $D_{k}$ is defined as

$$
V\left(D_{k} \mid D_{0}\right)=\max \left\{\left.V\left(D_{q} \mid D_{0}\right)\right|_{\substack{\tau_{0}=\tau_{s}\left(1-\delta_{v}\right) \\ \tau^{\tau}=\tau_{s}}},\left.V\left(D_{q} \mid D_{0}\right)\right|_{\substack{\tau_{0}=\tau_{s}\left(1+\delta_{v}\right) \\ \tau^{*}=\tau_{s}}}\right\},
$$

where $V\left(D_{q} \mid D_{0}\right)$ is variance of the dispersion estimate $D_{q}$, synthesized by the likelihood method, on the assumption that signal duration (15) is known inaccurately and is equal to $\tau^{*}\left(\tau^{*} \neq \tau_{0}\right)[7]$ :

$$
V\left(D_{q} \mid D_{0}\right)=2 E_{N}^{2} \int_{0}^{\infty}\left(x-q_{0}\right)\left[1-F_{S}^{*}(x) F_{N}^{*}(x)\right] d x+D_{0}^{2}
$$

In Eq. (24) we state that 


$$
\begin{gathered}
F_{S}^{*}(x)=\Psi_{0}\left[\zeta_{S}(x), m_{S}\right], \\
\zeta_{S}(x)=(\sqrt{\mu} / \sigma)\left[x\left(1+\delta_{\tau}\right)-q_{0}\left(1+\min \left(0 ; \delta_{\tau}\right)\right)\right], \\
\sigma^{2}=\left\{\begin{array}{c}
\left(1+q_{0}\right)^{2}\left(1+\delta_{\tau}\right), \delta_{\tau}<0 ; \\
\left(1+q_{0}\right)^{2}+\delta_{\tau}, \quad \delta_{\tau}>0 ;
\end{array} m_{S}=\left|\delta_{\tau}\right|\left\{\begin{array}{l}
1 /\left(1+\delta_{\tau}\right), \delta_{\tau}<0 ; \\
1 / \sigma^{2}, \quad \delta_{\tau}>0 ;
\end{array}\right.\right. \\
\Psi_{0}(u, \rho)=\int_{-\infty}^{u} \Phi\left[\frac{u-x(1-\rho)}{\sqrt{\rho(2-\rho)}}\right] \exp \left(-\frac{x^{2}}{2}\right) \frac{d x}{\sqrt{2 \pi}}- \\
\rho_{2 u} \exp \left(-\frac{u^{2}}{2}\right) \Phi\left(u \sqrt{\frac{\rho}{2-\rho}}\right)-\frac{\sqrt{\rho(2-\rho)}}{2 \pi} \exp \left(-\frac{u^{2}}{2-\rho}\right), \\
F_{N}^{*}(x)=\left\{\begin{array}{c}
\exp \left\{\frac{m x \sqrt{\mu}}{\sqrt{2 \pi\left(1+\delta_{\tau}\right)}} \exp \left[\begin{array}{c}
x^{2} \mu\left(1+\delta_{\tau}\right) \\
2
\end{array}\right],\right. \\
x \geq 1 / \sqrt{\mu\left(1+\delta_{\tau}\right)} ; \\
0,
\end{array}\right.
\end{gathered}
$$

$m=\left(\Lambda_{2}-\Lambda_{1}\right) / \tau_{0}$, and $\delta_{\tau}=\left(\tau^{*}-\tau_{0}\right) / \tau_{0}$ is a relative detuning on the pulse duration.

For the probability density $w\left(\tau_{m} \mid \tau_{0}\right)$ of the duration estimate $\tau_{m}$ in the measurer with the continuous change of current values of the estimated parameters in terms of the results [9] and by analogy to [3], we have

$$
w\left(\tau_{m} \mid \tau_{0}\right)=\int_{\Lambda_{1}}^{\Lambda_{2}} w_{2}\left(\lambda_{m}, \tau_{m} \mid \lambda_{0}, \tau_{0}\right) d \lambda_{m}
$$

where

$$
\begin{gathered}
w_{2}\left(\lambda, \tau \mid \lambda_{0}, \tau_{0}\right)= \\
=\frac{w_{1}\left[\lambda-\lambda_{0}-\left(\tau-\tau_{0}\right) / 2\right] w_{1}\left[\lambda_{0}-\lambda+\left(\tau_{0}-\tau\right) / 2\right]}{\int_{\Lambda_{1}}^{\Lambda_{2} \int_{T_{1}}^{\mathrm{T}_{2}}} w_{1}\left[\lambda-\lambda_{0}-\left(\tau-\tau_{0}\right) / 2\right] w_{1}\left[\lambda_{0}-\lambda+\left(\tau_{0}-\tau\right) / 2\right] d \lambda d \tau},
\end{gathered}
$$

and

$$
\begin{gathered}
w_{1}(x)=\frac{2}{\tau_{0}} \begin{cases}z_{1}^{2} w\left(2 z_{1}^{2} x / \tau_{0}, 1 / R\right), & x \geq 0 ; \\
z_{2}^{2} w\left(2 z_{2}^{2} x / \tau_{0}, R\right), & x<0 ;\end{cases} \\
z_{1}^{2}=\frac{\mu}{q_{0}^{2}}\left[q_{0}-\ln \left(1+q_{0}\right)\right]^{2}, z_{2}^{2}=\frac{\mu}{q_{0}^{2}}\left[\left(1+q_{0}\right) \ln \left(1+q_{0}\right)-q_{0}\right]^{2}, \\
R=\left(1+q_{0}\right)\left[\left(1+q_{0}\right) \ln \left(1+q_{0}\right)-q_{0}\right] /\left[q_{0}-\ln \left(1+q_{0}\right)\right], \\
w(x, u)=\Phi(\sqrt{|x| / 2})-1+[(2+u) / u] \exp \left[|x|(1+u) / u^{2}\right] \times \\
\times\{1-\Phi[\sqrt{|x| / 2}(2+u) / u]\} .
\end{gathered}
$$

Then, under the specified prior probability density $w_{p r}\left(\tau_{0}\right)$ of the parameter $\tau_{0}$, unconditional bias and variance of the estimate $\tau_{k}$ (19) can be found from Eq. (2), substituting $w_{p r}\left(\tau_{0}\right)$ and $w\left(\tau_{m} \mid \tau_{0}\right) \quad$ (25) for $w_{p r}\left(1_{0}\right)$, $w\left(1_{m} \mid 1_{0}\right)$, respectively.

To find out the experimental estimate characteristics, the statistical computer modeling of functional $M(\lambda, \tau)$ (18) was carried out. For reduction of computational burden it was supposed that the band limitedness condition of a kind $\vartheta \gg \Omega$ for the process $\xi(t)$ is satisfied. It allows to use representation of the filter response $y(t)$ (18) through its low-frequency quadratures [5] and to form sufficient statistics $M(\lambda, \tau)(18)$ as the sum of the two independent random processes:

$$
\begin{gathered}
M(\lambda, \tau)=\frac{1}{2}\left[M_{1}(\lambda, \tau)+M_{2}(\lambda, \tau)\right], M_{j}(\lambda, \tau)=\int_{\lambda-\tau / 2}^{\lambda+\tau / 2} y_{j}^{2}(t) d t \quad(26) \\
y_{j}(t)=\int_{-\infty}^{\infty} x_{j}\left(t^{\prime}\right) h_{0}\left(t-t^{\prime}\right) d t^{\prime}, x_{j}(t)=s_{j}(t)+n_{j}(t), j=1,2 .
\end{gathered}
$$

Here $s_{j}(t)=\xi_{j}(t) I\left[\left(t-\lambda_{0}\right) / \tau_{0}\right], \xi_{j}(t) n_{j}(t)$ are statistically independent centered Gaussian random processes with the spectral densities $G_{0}(\omega)=\left(2 \pi D_{0} / \Omega\right)(\omega / \Omega)$ and $N_{0}$, respectively, while the spectrum $H_{0}(\omega)$ of the function $h_{0}(t)$ satisfies a condition $\left|H_{0}(\omega)\right|^{2}=I(\omega / \Omega)$.

During modeling within the interval $\left[\tilde{\Lambda}_{1}, \tilde{\Lambda}_{2}\right]$, $\tilde{\Lambda}_{1,2}=\Lambda_{1,2} / \tau_{s}$, with discretization step $\Delta \tilde{t}$ in normalized time $\tilde{t}=t / \tau_{s}, \quad \tilde{y}_{j n}$ samples were formed of normalized random process realizations $\tilde{y}_{j}(\tilde{t})=y_{j}(t) \sqrt{\tau_{s} / N_{0}}, j=1,2$ (26). With Eqs. (27) In mind, we can achieve the step approximation of the normalized sufficient statistics $\tilde{M}\left(\tilde{\lambda}, \tilde{\tau}_{i}\right)=M\left(\lambda, \tau_{i}\right) / N_{0} \quad(18)$, presented as:

$$
M\left(\tilde{\lambda}, \tilde{\tau}_{i}\right)=\frac{\Delta \tilde{t}}{2} \sum_{n=N_{\min }}^{N_{\max }}\left(\tilde{y}_{1 n}^{2}+\tilde{y}_{2 n}^{2}\right)
$$

Here $N_{\min }=\operatorname{int}\left\{\left(\tilde{\lambda}-\tilde{\tau}_{i} / 2\right) / \Delta \tilde{t}\right\}, N_{\max }=\operatorname{int}\left\{\left(\tilde{\lambda}+\tilde{\tau}_{i} / 2\right) / \Delta \tilde{t}\right\}$, $\tilde{\lambda}=\lambda / \tau_{s}$ represent normalized current value of time delay; $\tilde{\tau}_{i}=\tau_{i} / \tau_{s}$ is normalized duration to which the $\mathrm{i}$-th measurer channel is tuned; and $\operatorname{int}\{\cdot\}$ is integer part.

With $\Delta \tilde{t}=0,05 / \mu_{s}$, the mean square error of the step approximation (27) of a continuous realization (26) did not exceed $10 \%$. Samples of processes $y_{j n}, j=1,2$ were formed in terms of the sequence of independent Gaussian random numbers by a moving summation method [5]:

$$
\tilde{y}_{j n}=\frac{1}{\sqrt{\Delta \tilde{t}}} \sum_{m=n-p}^{n+p} \alpha_{i m} H_{n m}+\sum_{m=\max \left(m_{\min }, n-p\right)}^{\min \left(m_{\max }, n+p\right)} \quad \xi_{j m} H_{n m},
$$




$$
\xi_{j m}=\frac{1}{\pi} \sqrt{\frac{q_{0}}{\Delta \tilde{t}}} \sum_{n=0}^{2 p} H_{n p} \beta_{j n+m+1}
$$

Here $m_{\min }=\operatorname{int}\left\{\left(\tilde{\lambda}_{0}-\tilde{\tau}_{0} / 2\right) / \Delta \tilde{t}\right\}, m_{\max }=\operatorname{int}\left\{\left(\tilde{\lambda}_{0}+\tilde{\tau}_{0} / 2\right) / \Delta \tilde{t}\right\}$, $\tilde{\lambda}_{0}=\lambda_{0} / \tau_{s}, \tilde{\tau}_{0}=\tau_{0} / \tau_{s}, \alpha_{j m}, \beta_{j m}$ are independent Gaussian random numbers with zero expectations and unit dispersions, $H_{n m}=\sin \left[2 \pi \mu_{s} \Delta \tilde{t}(n-m)\right] /[\pi(n-m)]$.

In the sums (28) number of summands corresponds to the value $p=100$. It provides a relative deviation of the generated sample dispersion from the modeled process dispersion to be no more than $5 \%$. Formation of independent Gaussian numbers with parameters $(0,1)$ was carried out following Eqs. (13).

In confinement with the Eqs. (26)-(28), realization of the process $\tilde{M}\left(\tilde{\lambda}, \tilde{\tau}_{i}\right)$ was produced, and normalized estimates $\tilde{\lambda}_{k}=\lambda_{k} / \tau_{s}, \quad \tilde{\tau}_{k}=\tau_{k} / \tau_{s}, \quad q_{k}=D_{k} / E_{N} \quad$ were defined according to Eq. (19) and their variances were found.

In Figs. 3-5 some results of statistical modeling are presented where corresponding theoretical dependences are shown also. Each experimental value was received as a result of processing of not less than $10^{4}$ realizations $\tilde{M}\left(\tilde{\lambda}, \tilde{\tau}_{i}\right)$, with $\mu_{s}=150, q_{0}=1, \tilde{\Lambda}_{1}=0, \tilde{\Lambda}_{2}=\tilde{m}=17$, $\tilde{\lambda}_{0}=\left(\tilde{\Lambda}_{2}+\tilde{\Lambda}_{1}\right) / 2, w_{p r}\left(\tau_{0}\right)$ (10). Thus with probability of 0.9 confidence intervals boundaries deviate from experimental values no more than for $10 \ldots 15 \%$. In Fig. 3 continuous lines represent dependences of the normalized variance $\tilde{V}_{\lambda}=12 V\left(\tilde{\lambda}_{k} \mid \tilde{\lambda}_{0}\right) / \tilde{m}^{2}$ of estimate $\tilde{\lambda}_{k}$ from parameter $\delta(20)$. Curves 1 are calculated via formulae (21), (22), with $v=2,2$ $-v=3,3-v=5$. By squares, crosses and rhombuses the experimental values of estimate variance $\tilde{V}_{\lambda}$ are designated for $v=2,3$ and 5. In Fig. 4, 5 the similar dependences are presented of maximum variance $\tilde{V}_{q}=V\left(q_{k} \mid q_{0}\right)$ of estimate $q_{k}$ and of unconditional variance $\tilde{V}_{\tau}=V\left(\tilde{\tau}_{k}\right)$ of estimate $\tilde{\tau}_{k}$ plotted by formulae (23), (24) and (2), (25), respectively. Designations in Fig. 4, 5 are the same as in Fig. 3.

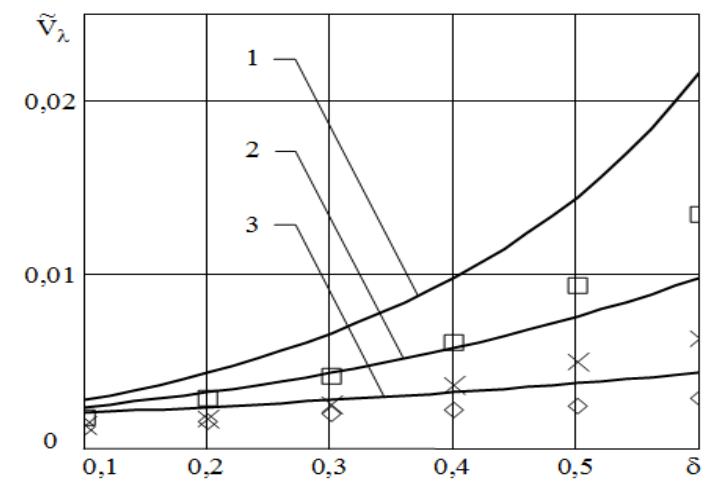

Figure 3. Normalized variance of time delay estimate of random radio pulse.

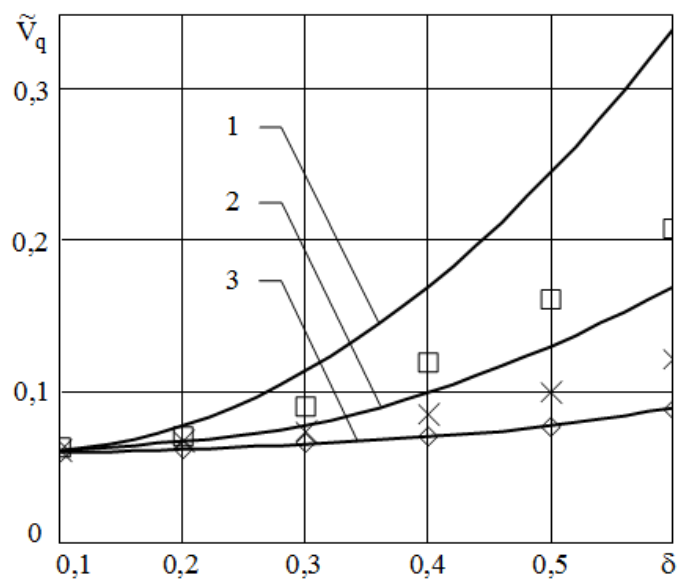

Figure 4. Normalized variance of dispersion estimate of random radio pulse.

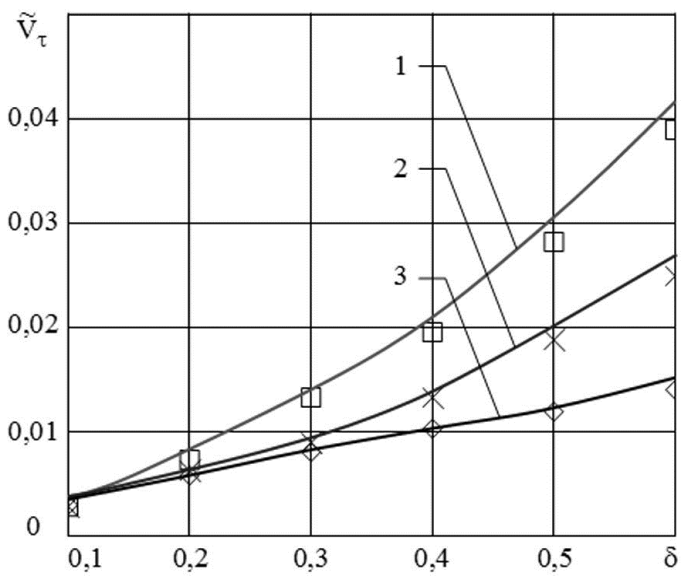

Figure 5. Normalized variance of duration estimate of random radio pulse.

As follows from Figs. 3-5 and the conducted modeling, the theoretical dependences (21)-(24) for the maximum variances of estimates $\lambda_{k}$ and $D_{k}$ can be used for practical calculations, if , at least, $\mu_{s} \geq 150, z_{s} \geq 1, v \geq 2$; and their matching with the values of real variance improves with the increasing $v$ and decreasing $\delta$. So, with $\delta \leq 0.6, v \geq 2$ maximum variances of estimates $\lambda_{k}$ and $D_{k}$ no more than 2 times higher than the true ones, and when $\delta \leq 0.3, v \geq 3$, they are no more than 1.1 times higher. The formulae (2), (25) for unconditional variance of signal duration estimate $\tau_{k}$ adequately describes the corresponding experimental values for $z_{s} \geq 1$ and $v \geq 2$, and they are substantially more accurate than the already known formulae, describing the multichannel reception signal parameters estimates characteristics [2, 3].

\section{Conclusion}

In order to define the multichannel measurer characteristics, it is possible to use the expressions for the unconditional bias and variance of a decided estimate, found in the present work. The introduced expressions are correct for any type of signal and its estimated parameter, and they also have essentially greater precision in comparison with 
the formulae for accuracy characteristics of multichannel reception, adduced in the known literature [2, 3]. The conducted computer experiments show that the received new formulae adequately describe experimental data in a wide range of output SNR values and with any number of measurer channels.

\section{Acknowledgements}

The reported study was supported by Russian Foundation for Basic Research, research projects No. 13-08-00735a, 13-08-97538.

\section{References}

[1] A.P. Trifonov, Yu.S. Shinakov, Joint Discrimination of Signals and Estimation of their Parameters against Background [in Russian]. Moscow: Radio i Svyaz', 1986.

[2] E.I. Kulikov, A.P. Trifonov, Estimation of Signal Parameters against Hindrances [in Russian]. Moscow: Sovetskoe Radio, 1978.

[3] A.P. Trifonov, V.K. Buteiko, "Choice of channels number and signal parameter estimate against hindrances" [in Russian],
Radio engineering (Moscow), 1981, vol. 36, no. 4, p. 56-58.

[4] A.P. Trifonov, V.I. Parfenov, D.V. Mishin, "Optimum reception of a signal with unknown duration against the background of white noise", Radiophysics and Quantum Electronics, 1997, vol. 40, no 12, pp. 1031-1038.

[5] V.V. Bykov, Numerical Modeling in Statistical Radio Engineering [in Russian]. Moscow: Sovetskoe Radio, 1971.

[6] Luc Devroye, Non-uniform Random Variate Generation. Springer-Verlag, 1986.

[7] O.V. Chernoyarov, "The statistical analysis of the noise-stable processing algorithm of the stochastic impulse signal with unknown parameters", Proceedings of the III International Conference "Antennas, Radiocommunication Systems and Means” (ICARSM'97). - Voronezh: VSU, 1997, vol. 2, pp. 185-195.

[8] A.P. Trifonov, A.V. Zakharov, O.V. Chernoyarov, "Threshold characteristics of the quasiprobable estimate of the arrival time of a random radio pulse", Radioelectronics and Communications Systems, 1998, vol. 41, no 10, pp. 12-19.

[9] A.P. Trifonov, A.V. Zakharov, "A theoretical and numerical study of the estimators for the parameters of a random signal with unknown moments of appearance and disappearance", Journal of Communications Technology and Electronics, 1996, vol. 41, no 10, pp. 907-913. 